\title{
A RECORDING CHRONOGRAPH FOR THE INVERSE RATE METHOD OF THERMAL ANALYSIS.
}

\author{
By H. J. French.
}

ABSTRACT.

This report describes an apparatus designed for direct plotting of curves for the inverse rate method of thermal analysis in which the time interval required for a definite temperature change and the temperature are the coordinates. The fundamentals of its construction and operation are described and examples of typical curves produced are included. There is also given a brief discussion of the advantages obtained by its use, principally in reducing the time required for determining transformations in metals with equal or greater accuracy than when using ordinary types of chronographs.

\section{CONTENTS.}

Page.

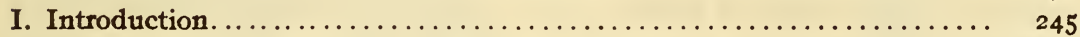

II. Description of the direct plotting chronograph............... 247

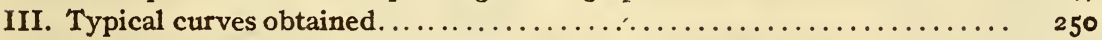

IV. Discussion of constructional features of the instrument........... 253

\section{INTRODUCTION.}

One of the most useful methods of thermal analysis, whereby very small arrests may be detected in the heating and cooling of metals, is that known as the inverse rate method in which the time interval required for a definite temperature change is plotted against the temperature. Its limited application has been due largely to the expensive equipment required but in part to the lack of suitable recording apparatus for direct plotting of curves in order to eliminate the long and tedious processes involved in their preparation from data obtained with the usual types of chronographs.

A special recording instrument for such work, designed by Rosenhain, ${ }^{1}$ has been in use for some time at the National Physical Laboratory in England, but so far as is known by the author, this instrument has not been used in the United States and no others of a similar nature are available.

1 W. Rosenhain: Journ. Inst. Metals, 13, p. I60; 1915. 
The equipment and procedure employed heretofore at the $\mathrm{Bu}$ reau of Standards has already been described in detail, ${ }^{2}$ and is essentially as follows:

A small slotted specimen of the test metal, mounted on a platinum-9o platinum, ro rhodium-thermocouple and placed in an evacuated silica tube, is raised or lowered at a definite and predetermined rate in a tubular electric resistance furnace which is mounted in a vertical position. The platinum heating coil used is restricted to the upper end of the furnace, which has a relatively large thermal capacity compared to the sample being heated, and is so constructed that a gradual decrease in temperature is obtained from top to bottom. Under such conditions the rate of temperature change to which the metal is subjected is dependent upon the rate at which the specimen is moved up or down in the heating unit. This is controlled by weights acting against an adjustable flow of oil moving a plunger in a cylinder. The flow of oil is regulated by a calibrated needle valve, and the plunger is connected to the tube which contains the test specimen.

The method employed with this equipment consists in recording on the usual type of drum chronograph the time required for a definite temperature change in the specimen, while temperature measurements are taken by means of the thermocouple and a dial potentiometer. The chronograph records are subsequently "counted" and the curve plotted by hand with time intervals as abscissas and mean temperatures during the intervals as ordinates.

In order to eliminate part of the expensive equipment required and at the same time reduce somewhat the work of preparing the graphs, Merica ${ }^{3}$ proposed the use of stop watches to measure time intervals. This method eliminates the "counting" of chronograph records, but it is still necessary to plot curves by hand, and the ratio of time consumed in this operation to that actually employed in making observations remains quite large.

Obviously, the only satisfactory method for rapid production of inverse-rate curves, particularly those covering a wide range in temperature when the plotting of 500 to $\mathrm{r}, 000$ points may be required, as shown in Figure 7 , is by the use of a direct plotting chronograph, and to meet this condition the apparatus shown in Figure I was designed. Its use for over four months in determining transformations in various carbon and alloy steels under 
Technologic Papers of the Bureau of Standards, Vol. 17

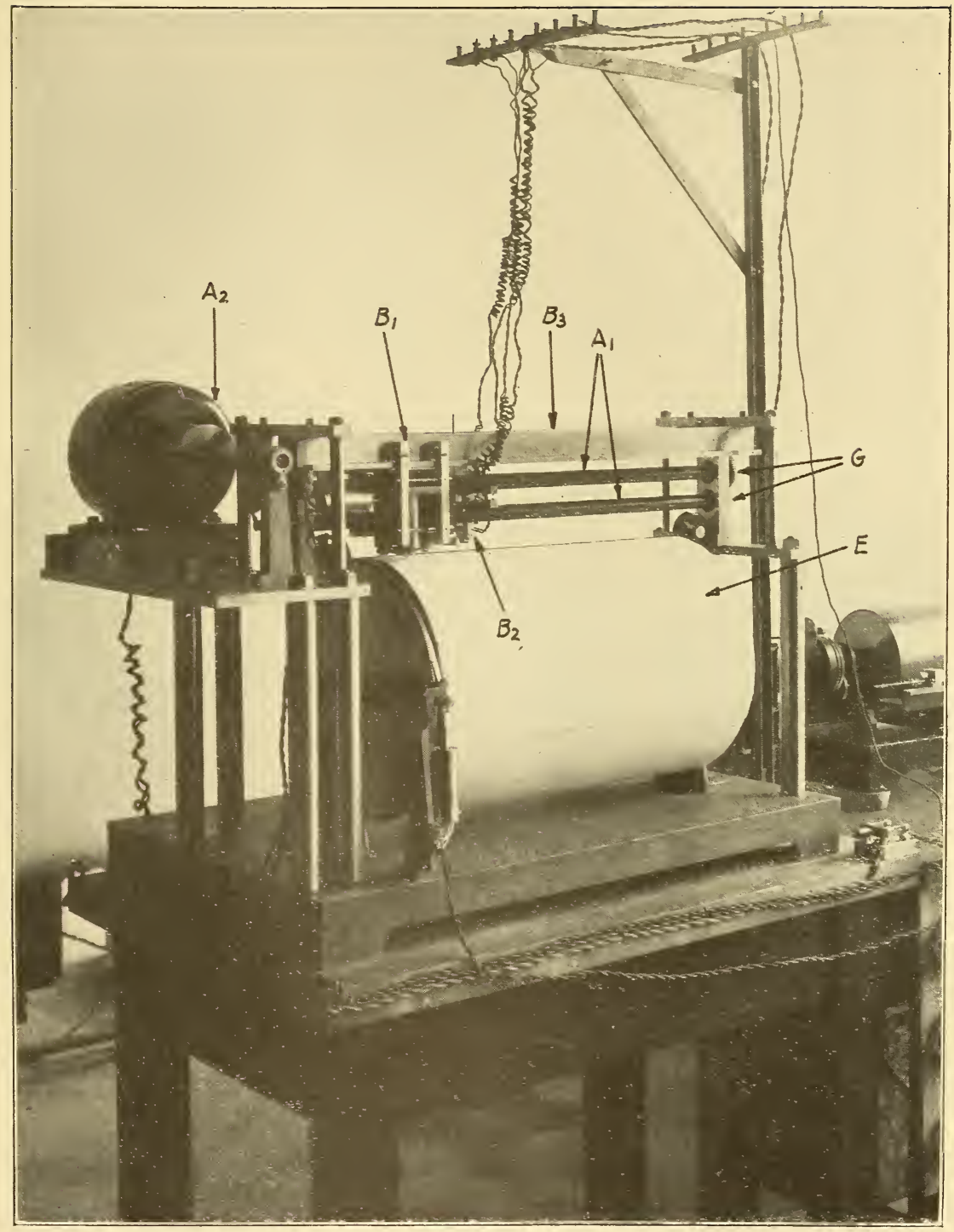

FiG. I.-Direct plotting chronograph for the inverse rate method of thermal analy'sis. 
Technologic Papers of the Bureau of Standards, Vol. 17

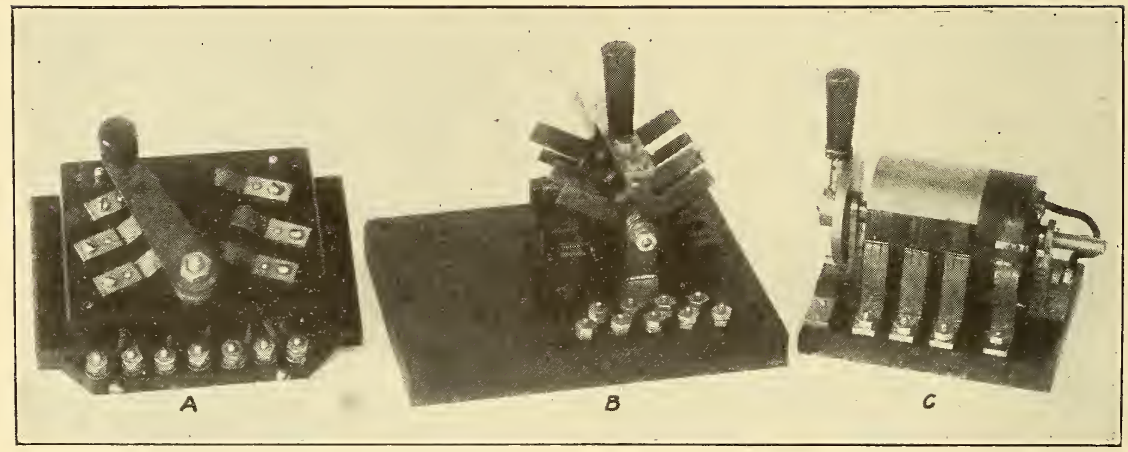

FIG. 2.-Special switches used. 
varying thermal treatments has shown it to be well suited for both routine and research work and has resulted in a marked saving in time and effort with increased accuracy in the results obtained.

\section{DESCRIPTION OF THE DIRECT PLOTTING CHRONO- GRAPH.}

The important parts of the apparatus, in addition to suitable mountings, are $(a)$ propelling screws and motor drive, $(b)$ carriages with pens and contact arms, $(c)$ special switch, $(d)$ electromagnets, and $(e)$ drum carrying the record paper.

Two screws, shown at $A_{1}$ in Figure I, are driven at constant speeds in opposite directions by motor $A_{2}$, on which is mounted a suitable governor. The upper screw brings both carriages forward when engaged by their contact arms, while the lower one is used for return to their starting points or "zero positions." By means of the gear reduction shown at $G$ and variation in the pitch of the two screws the return of the carriages is made ten times as fast as their forward movement.

Two carriages (shown at $B_{1}$ and $B_{2}$ in Fig. I) are used. Each carries a pen and contact arms for engaging the propelling screws and runs on an overhead track, which in each case is grooved to eliminate side play.

In order to record continuously time intervals, it is necessary that one carriage always be at its zero position or "base," ready to start forward, when the other is stopped, a record made of its position and return to its starting point begun. To accurately measure such intervals requires very rapid reversal of the system, and this is carried out with the aid of a special double-throw "gang" switch controlling two sets of electromagnets. The mechanism of reversal is probably best illustrated diagramatically, a definite set of conditions first being assumed.

As indicated in Figure 3, carriage $F$ is moving away from its zero position (forward), the contact arm $A_{1}$ being engaged to the upper propelling screw. At the same time carriage $R$ is returning to base at ro times the speed at which $F$ is going forward. It will also be noted from Figure 3 that the "reversing magnets" $K_{1}$ and $K_{2}$ are so arranged that when carrying current they throw the contact arms of both carriages against the "return screw," while when no current passes through their respective circuits the springs $S_{1}$ and $S_{2}$ hold these arms in contact with the "forward screw." It is 
therefore possible by breaking the circuit $k_{2}$ and closing $k_{1}$ to reverse the system, so that carriage $R$ moves forward from its base and $F$ returns. This change may readily be handled by a doublethrow switch, the moving arm of which travels through a small arc. By adding to it the necessary contact points it is possible to excite additional magnets operating auxiliary equipment, including the recording pens, which will give a record of carriage or pen positions at any instant that reversal of the system is desired.

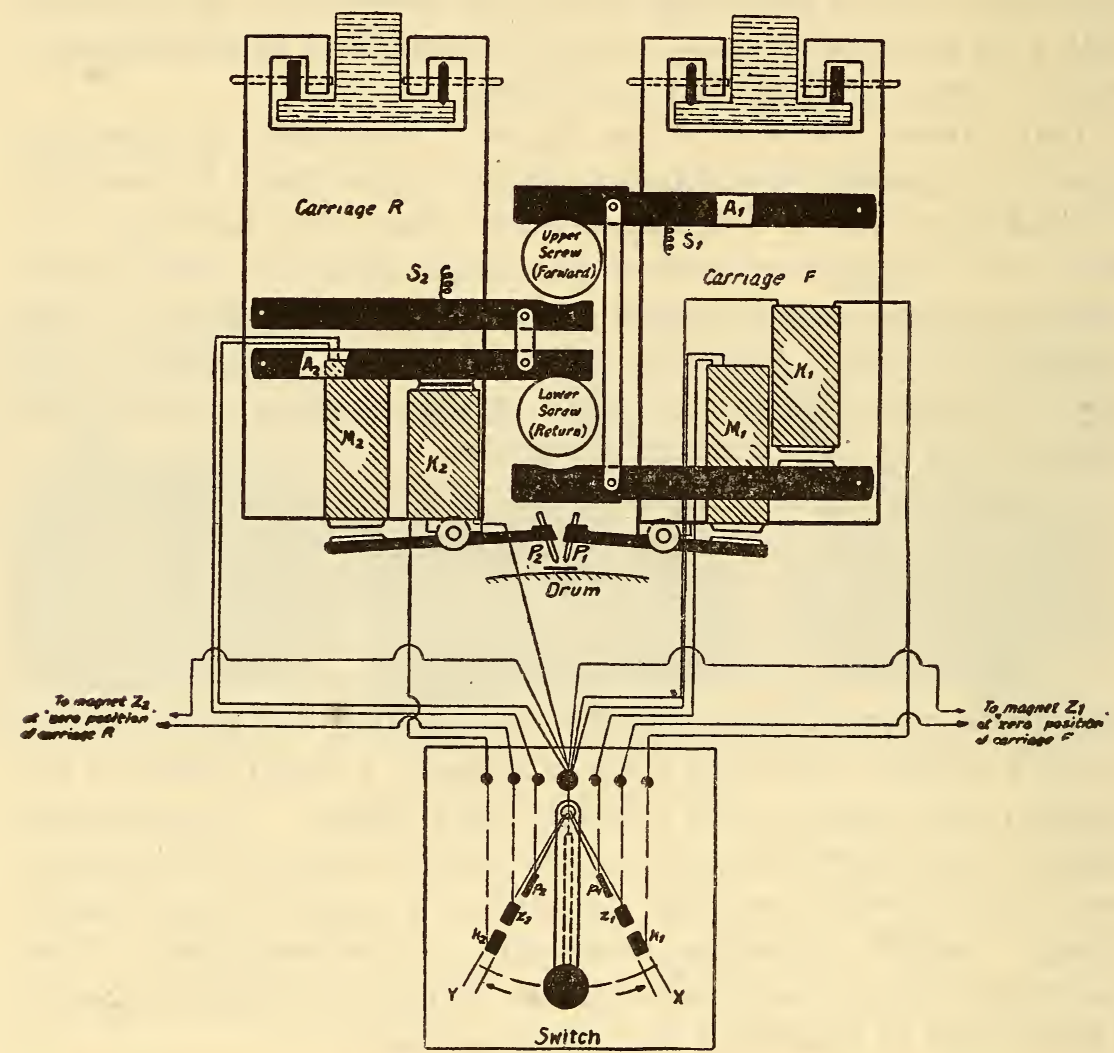

FIG 3.-Diagram showing fundamentals of design and operation of the direct plotting chronograph.

The return of the carriages is quite rapid, so that the stops placed at their base positions are subjected to considerable impact. Tapered pins are used to raise their contact arms into a neutral position to prevent "stripping" the threads of the screws, and there is therefore a decided tendency for the carriages to rebound. To avoid this and insure identical zero pen positions after each return, additional magnets are placed at the base stops and hold the carriages in place until they again start forward. Thus, six 
magnets are controlled by the special double-throw "gang" switch which is thrown from $X$ to $Y$ or $Y$ to $X$, depending upon its position at the instant the operator desires reversal.

As the moving arm of the switch travels from $Y$ to $X$, for example, pen $P_{2}$ is depressed and a record of its base position obtained. Because of the small width of contacts controlling the pen magnets the circuit $p_{2}$ is quickly broken and the pen raised, thereby preventing it being dragged along the paper. Simultaneously circuits $k_{2}$ and $z_{2}$ are opened, resulting in the release of the carriage $R$ by the base magnet $Z_{2}$ at the same time that the contact arm is released by magnet $K_{2}$ and pulled by spring $S_{2}$ against the forward screw. When the moving arm of the switch reaches contacts $z_{1}$ and $k_{1}$ the zero magnet for carriage $F$ is excited, and simultaneously the contact arm is thrown from forward into reverse. At the same instant the pen $P_{1}$ is depressed by its magnet and then quickly raised by the breaking of the circuit at $p_{1}$. This en tire cycle of change requires less than onefifth of a second under ordinary conditions.

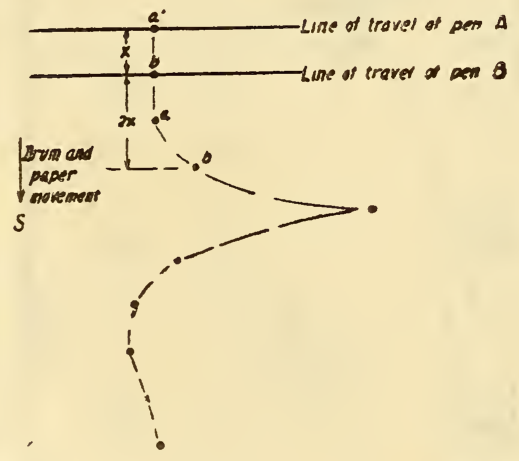

FIG. 4.-Relation of drum movement to pen positions.

To plot time intervals as a function of temperature by means of the mechanism just described does not offer any special difficulties. Instead of a drum rotating at a constant and predetermined speed, such as is employed with the usual type of recording chronograph, one is required which can be moved a fixed distance whenever desired. If the distance between the line of travel of the two pen points is equal to $x$ (Fig. 4) and the first time interval recorded in any run is made with pen $B$, then it is not necessary to move the drum carrying the paper until after $A$ has recorded the second interval. It should then be moved in the direction $S$ a distance equal to $2 x$. This motion brings a new point, $b^{\prime}$, under the pen $B$ and a new point, $a^{\prime}$ under the pen $A$, the lateral distance between $b$ and $b^{\prime}$ and between $a$ and $a^{\prime}$ being $2 x$. The proper motion of the drum can readily be effected by a ring gear on the periphery of the drum and a simple mechanical device operated by an electromagnet as shown in Figure I. As the two pens alternate in recording time intervals and rotation of the drum is only required after each second 
interval, control of the latter may be added to the special "gang" switch by placing an additional contact point on that side where the carriage of pen $B$ of Figure 3 is thrown from forward into re-

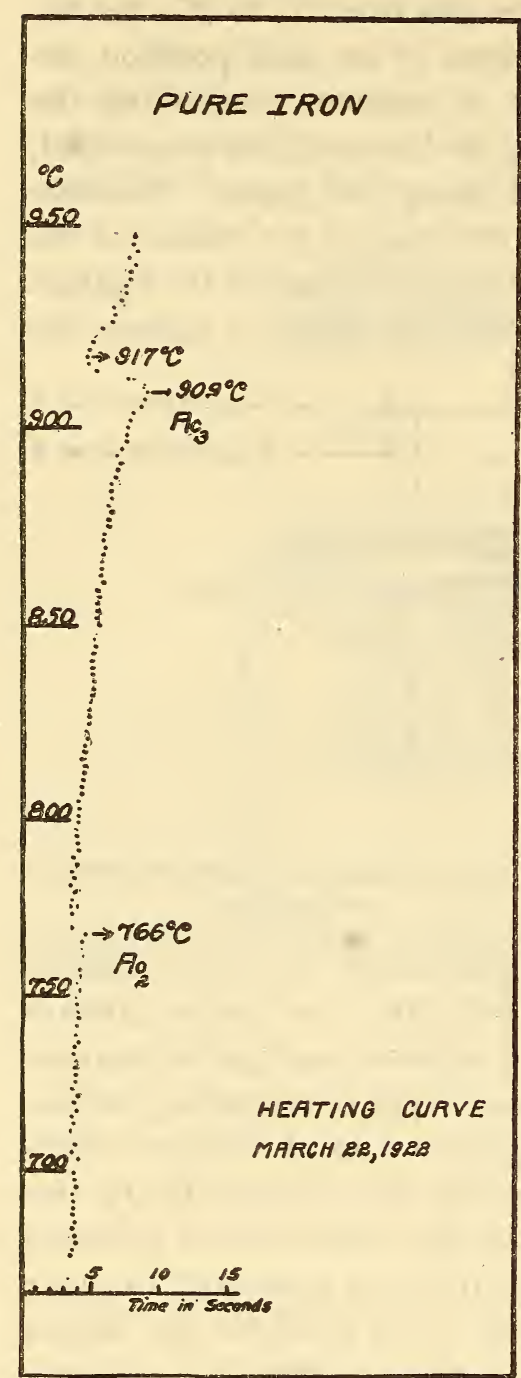

FIG. 5.-Heating curve of pure iron obtained with the direct plotting chronograph.

About 25 minutes required for the "run." Reproduction about one-fourth size. verse. Thus, time intervals are recorded as abscissas by the movement of pens along the axes of the propelling screws, while temperatures are recorded as ordinates by means of the intermittent rotation of the drum.

The procedure employed with this special chronograph is similar to that used with the ordinary type. The operator sets the potentiometer at successive values of emf, differing by equal increments, usually 0.02 millivolt, but instead of pressing a contact key at the exact instant at which the galvanometer coil passes through its null position throws the special switch, which reverses the entire system.

\section{TYPICAL CURVES OBTAINED.}

Reproduction of typical curves obtained by the use of the apparatus is given in Figures 5, 6 , and 7. Relatively minute thermal arrests, such as the $A_{2}$ transformation in pure iron (Fig 5), which are not readily observed except when using accurate methods, are clearly shown. The characteristics of different transformations are also well preserved, as is illustrated in Figure 7, where both "sharp" and "rounded points" appear. Likewise equally good results are obtained when using rapid (Fig. 5) or slow (Fig. 7) rates of temperature change. 
The curves are not produced automatically and are therefore still dependent upon the careful work of an operator. They are, however, less susceptible to errors arising purely from the "human element" than are curves prepared by methods employed in hand plotting, as the "counting" of chronograph sheets containing several hundred observations, and the placing of values so obtained upon finely divided cross-section paper demands most painstaking and careful work to prevent the introduction of errors.

Long and short readings, resulting from delay in throwing the switch as the galvanometer passes its null position, are not eliminated but are definitely shown by reason of the fact that the resulting points will be at equal distances and on opposite sides of the true curve. One or two examples of this are contained in Figure 7. However, errors introduced in the plotting are avoided.

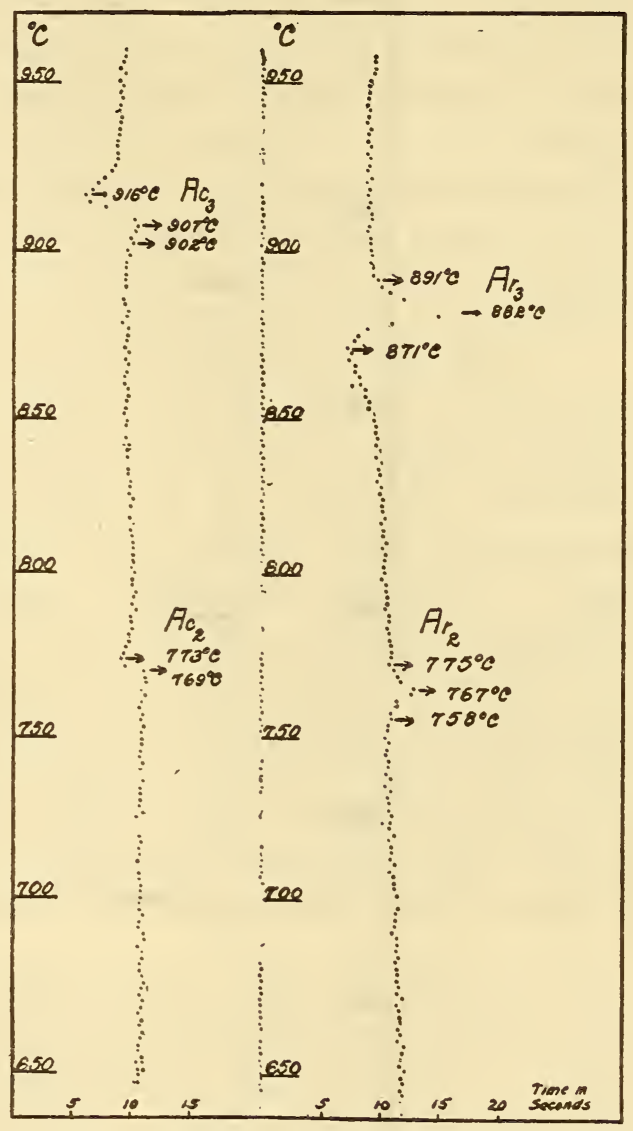

A desirable feature of FIG.6.-Heating and cooling curves of copper bearthe curves is the check obtained on the base position of both pens prior to reing steel.

C, 0.06 per cent; $\mathrm{Cu}, 0.24$ per cent. Reproduction about one-fifth size.

cording each time interval. This was inadvertently introduced with the use of the reversing switch and equipment previously described, but is of considerable value in preventing introduction of errors due to a changing base line brought about by loosened carriage or pen parts or other causes. 


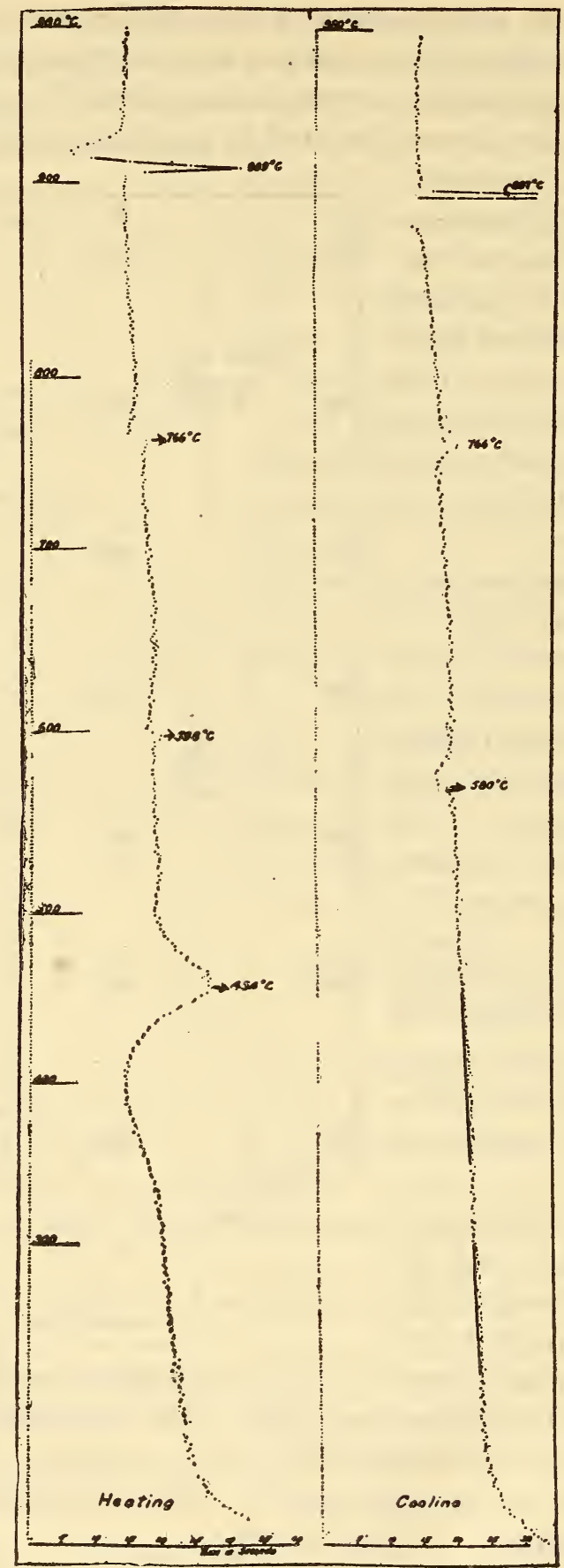

FIG. 7.-Heating and cooling curves of electrolytic iron remelted under an atmosphere of nitrogen in a zirconium crucible, and to which a small proportion of calcium has been added. 


\section{DISCUSSION OF CONSTRUCTIONAL FEATURES OF THE INSTRUMENT.}

Because of the varying magnitude of thermal transformations a high speed return of the pens is necessary. Usually long arrests are followed by very short intervals, and a condition may obtain where insufficient time is allowed for one carriage to return to its base position before it is again required to start forward to record a subsequent interval. In general, a ratio of $\mathrm{Io}$ to $\mathrm{I}$ is sufficient, though one or two cases have already been encountered where this was not so. However, it is questionable whether any additional information of value would be obtained by following completely such extremely large thermal effects, though they may readily be recorded by increasing somewhat the speed ratio referred to.

One of the important features of the equipment is the special switch, several types of which have been tried, as shown in Figure 2. Type A was used originally and was generally satisfactory except for the rapid burning of the contacts, which required considerable attention to insure satisfactory operation. A knife switch, such as type B, was tried, but, due to excessive arcing and inconvenience in operating it, was discarded. This type was replaced by $\mathrm{C}$, which proved to be the most desirable and has been adopted. It is recognized, however, that the early difficulties encountered are due to the relatively high current necessary for operating the magnets as originally constructed, and that by rewinding for lower current such undesirable effects would be largely eliminated.

The recording pens are different from those ordinarily employed. A two-color typewriter ribbon is stretched along the line of travel of the pens, which consist of small steel rods rounded at the ends and mounted in suitable holders. When depressed by their magnets, the pens strike the ribbon, which is close to the paper, leaving a small round dot. Thus, the use of fountain pens or other types containing liquids, which are not well suited to the service requirements in this instrument and which ordinarily require a little special attention, is avoided.

The original apparatus shown in Figure I was purposely made larger than necessary in order that adjustments and such changes as might be desired could more readily be made in the laboratory, but there is no reason why a second model should not be made smaller and portable. The width and diameter of the drum may be reduced. Also, the carriages, propelling screws, and many of 
the mountings are larger than required, but an adequate driving force must be retained, as constant speed of rotation of the screws is one of the essential features of satisfactory operation. Engaging the contact arms is accompanied by impact and results in sudden changes in load which must be absorbed by the motor and governor without noticeable speed change in the recording pens.

It is not necessary to restrict the use of the apparatus to the special furnace previously mentioned, as entirely satisfactory curves for ordinary purposes may be obtained with any heating unit in which a moderately uniform rate of temperature change can be produced, and under such conditions determination of transformations may be made in a very short time. The curve shown in Figure 4 was obtained in about 25 minutes after the test sample was prepared by heating it in air in a small and inexpensive resistance furnace.

Certain modifications or additions to the original apparatus shown in Figure I are now in progress. These include the installation of a simple mechanism. for moving the typewriter ribbon with the drum in order to distribute the wear resulting from pens repeatedly striking within a narrow zone; a shifting device for more readily changing the record from black to red, instead of interchanging the positions of spools carrying the two-color ribbon; a "base shift" for the drum, so that heating and cooling curves may be obtained side by side instead of as in the original apparatus, one over the other, unless the paper position was changed.

The instrument described serves the same purpose as that in use in the National Physical Laboratory, previously referred to. However, two pens are used, as suggested by O. F. Hudson, ${ }^{4}$ thereby obviating the necessity of providing compensation for the time required for a single pen to return to its base position. Also, the use of a typewriter ribbon and solid steel bars for producing the records gives much less trouble than any type of pen already devised for the use of liquids in producing curves by a series of detached markings, and the use of a special double-throw "gang" switch, which is not much more troublesome to operate than a key, has simplified the electrical control. It is believed the new instrument is more simply and therefore more cheaply constructed. 
The author takes pleasure in making acknowledgment to J. Ludewig, mechanician, instrument shop, Bureau of Standards, who contributed so largely to the success of this instrument by working out the mechanical details from incomplete drawings and sketches containing principally the fundamental features of design, and to Misses I. Wymore, assistant chemist, and M. Preble, senior aid, who have handled the laboratory work in connection with the trial of the apparatus.

WASHINGTON, November 8, 1922. 